\title{
DIAGNOSTIC DIFFICULTY AND STRATEGY FOR GALLBLADDER ADENOMYOMATOSIS
}

\author{
Michelli Daltro Coelho Ridolfi, MD.,Camila Mattje Backes, MD.,Thatiany Gracino de Marqui, MD \& Henrique \\ José Pereira de Godoy, MD
}

'Department of Surgery of Federal University of Mato Grosso-UFMT-Brazil

2 Physician University of Cuiaba-UNIC-Brazil

3.4 Physician Federal University o Mato Grosso-UFMT-Brazil

(Received on Date:26 th June 2020

Date of Revision\& Acceptance: $7^{\text {th }}$ July 2020

Date of Publish:1 ${ }^{\text {st }}$ September 2020)

Email : henriquegodoy95@gmail.com

\begin{abstract}
Background: The differential diagnosis of gallbladder adenomyomatosis is not always easy and, many times, the option is surgical. The aim of the present study was to report the diagnostic strategy for gallbladder adenomyomatosis in an asymptomatic older patient. Case presentation: A 76-year-old female patient was sent to the digestive tract surgery service of the Cuiabá Santa Casa Hospital in Brazil due to evidence of polyps in the gallbladder diagnosed by tomography during the follow-up of repeated urinary infections. The tomogram revealed irregular thickness with the densification of adipose planes of the fundus of the gallbladder without dilation of the bile ducts. Magnetic resonance cholangiopancreatography was performed for a better assessment of the gallbladder and biliary tree. The exam revealed heterogenous intraluminal structures in the fundus region of the gallbladder, with focal points of hypersignals in $\mathrm{T} 2$ and an area highlighted by the para-magnetic contrast medium measuring $2.0 \mathrm{~cm}$ along the longest axis and colonic diverticulosis with no signs of infection. Surgery was performed and the histopathological analysis revealed gallbladder adenomyomatosis. Conclusion: In cases of diagnostic doubt and considering the possibility of malignancy, the option should be surgery.
\end{abstract}

Keywords: Gallbladder adenomyomatosis, neoplasm, diagnosis, surgery 
Introduction

Gallbladder adenomyomatosis is an acquired benign abnormality in which the mucous epithelium hypertrophies together with the muscle layer, forming Rokitansky-Aschoff sinuses, which are saccular dilations of the mucosa. 1

The etiology and pathology have not been fully clarified. However, chronic inflammation of the gallbladder is known to be the precursor. There are hypotheses that this condition is the result of abnormal muscle contractions and consequent abnormal intraluminal pressure.1,2 It is classified based on the locus of installation as diffuse, segmental or localized.2

The prevalence ranges from 1 to $9 \%$ of the population, with a peak incidence after 50 years of age, affecting women more than men. Moreover, 50 to $90 \%$ of cases are associated with gallstones. 2,3

Although asymptomatic in most cases, this condition can be accompanied by abdominal pain or hepatic colic, very similar to cholecystitis. The diagnosis is performed using ultrasonography, which indicates an intraparietal pseudocystitis. ${ }^{2}$
Despite reports of cancer associated with gallbladder adenomyomatosis, treatment for asymptomatic cases is not indicted. In contrast, cholecystectomy is appropriate when there is intense pain with no explanation or when associated with gallstones. 3,4

The differential diagnosis is of extreme importance due to the possible evolution of the condition. This paper describes a case of a patient with gallbladder adenomyomatosis, identifying the indication criteria: symptoms, associated gallstones and the differential diagnosis of gallbladder neoplasm.

\section{Case report}

A 76-year-old female patient, retired, resident of the city of Cuiabá, Brazil, was sent to the digestive tract surgery service of the Cuiabá Santa Casa Hospital due to evidence of polyps in the gallbladder diagnosed by tomography during the follow-up of repeated urinary infections. The tomogram revealed irregular thickness with the densification of adipose planes of the fundus of the gallbladder and without dilation of the bile ducts (Figure 1). 


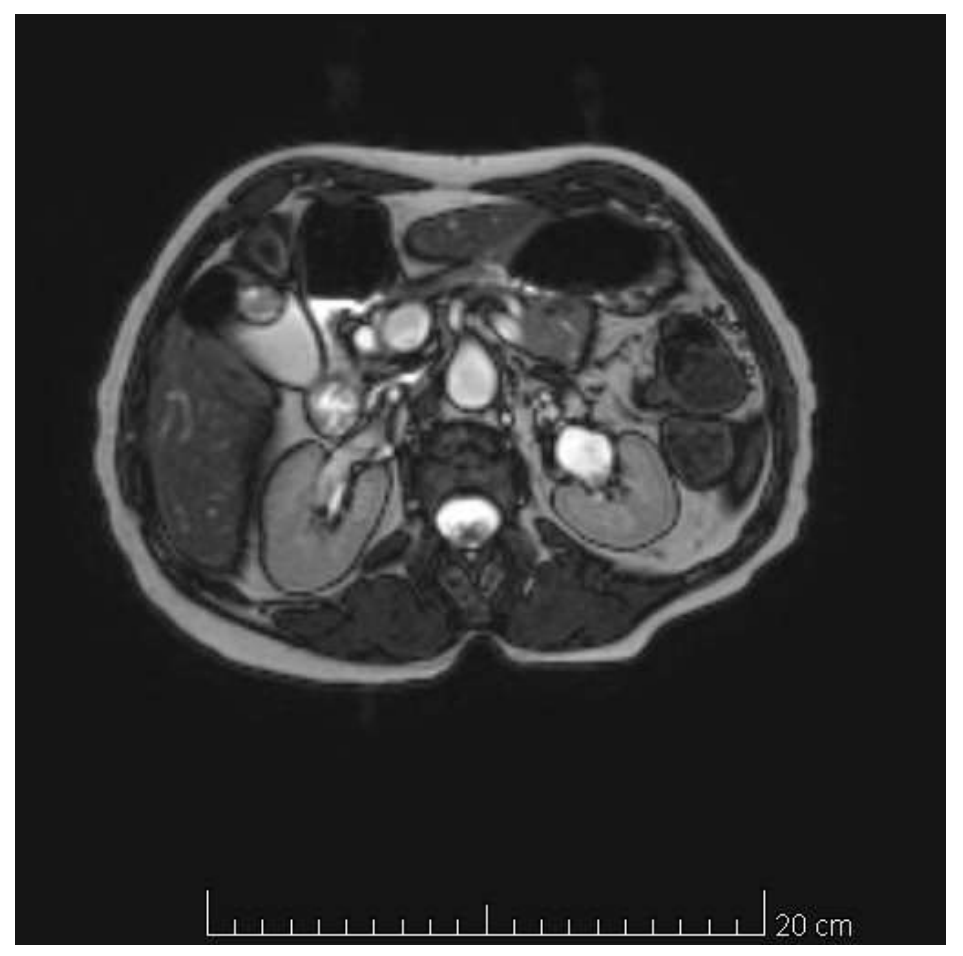

Figure 1: Preoperative tomogram.

The patient reported no abdominal pain in the upper right quadrant but noticed a sensation of fullness after meals unrelated to any dietary pattern. She reported no change in bowel movement pattern or characteristics of the stool, vomiting, nausea, anorexia, reflux or changes in appetite.

The physical examination revealed no abnormal findings, pain upon deep palpation, quantitative change in fluid noises or jaundice.

Due to the absence of suggestive signs and the patient's age, routine laboratory exams were requested for the evaluation of liver and kidney function. Moreover, magnetic resonance cholangiopancreatography was performed for a better assessment of the gallbladder and biliary tree. The exam revealed heterogenous intraluminal structures in the fundus region, with focal points of hypersignals in $\mathrm{T} 2$ and an area highlighted by the para-magnetic contrast medium measuring $2.0 \mathrm{~cm}$ along the longest axis and colonic diverticulosis with no signs of infection. The laboratory exams were returned wtih the following values for tumor markers: carcinoembryonary antigen of $1.56 \mathrm{ng}$ $\mathrm{mL}, \mathrm{CA} 19-9$ less than $2 \mathrm{U}-\mathrm{mL}$ and alphafetoprotein of $2.37 \mathrm{ng}-\mathrm{mL}$. All carcinogenic markers revealed a pattern of normality and all other laboratory results were normal.

Elective surgery (laparoscopic cholecystectomy) was scheduled. The surgery occurred with no complications and a biopsy was performed of the removed gallbladder. The histopathological analysis revealed gallbladder adenomyomatosis. 
adenomyomatosis is a precursor of adenocarcinoma

\section{Discussion}

This paper reports a case of an older, post-menopausal, white patient with imaging exams revealing thickening of the wall of the gallbladder associated with polyps, raising the hypothesis of a gallbladder neoplasm based on the risk factors and findings on the imaging exam.

The female sex has a two-to-six-fold higher incidence compared to males, especially in white post-menopausal women. Other risk factors include diabetes, chronic cholangitis, the use of anticonception medication and obesity. ${ }^{2}$

Gallbladder carcinoma is known to progress rapidly and has a poor prognosis, with a $28 \%$ five-year survival rate and survival estimated to be only 10 months when not diagnosed early, as occurs in most cases, since the initial imaging findings are discrete and the clinical condition is initially mild. In contrast, the finding in the patient reported herein was gallbladder adenomyomatosis, which has a benign course and good resolution. ${ }^{5}$

However, the two conditions are correlated. Although gallbladder adenomyomatosis is a non-inflammatory condition, it is strongly correlated with gallstones, which can lead to a chronic inflammatory process, the main risk factor for the development of local carcinoma. Moreover, several researchers, such as Nabatame et al., ${ }^{7}$ state that
The surgical indication for gallbladder adenomyomatosis or suggestive imaging findings is for cases in which moderate or severe symptoms are not controlled with clinical treatment. However, one must balance the weight of risk factors, the clinical condition of the patient and associated comorbidities that lead to a possible diagnosis of carcinoma, as in the present case, in which the patient had a polyp larger than $1 \mathrm{~cm}^{3}$

The origin of polyps may be adenoma, inflammatory, miscellaneous or cholesterol, the latter of which is the most prevalent. Polyps larger than $1 \mathrm{~cm}$ with no signs of necrosis or calcification are indicative of malignancy. Surgery is recommended for polyps larger than 1 $\mathrm{cm}$ and annual follow-up is indicated for those smaller than $1 \mathrm{~cm}$, independently of the symptoms. 8

In the case reported herein, despite the mild symptoms, we considered the fact that the patient had certain risk factors, such as her age, sex, post-menopausal condition, gallstones and polyps and the uncertainty of the polyps as either malignant at the onset of the disease or benign with a subtle evolution. Also considering the irregular thickening of the fundus of the gallbladder, the decision was made to perform laparoscopic cholecystectomy.

Even with the confirmation of the diagnosis by histopathological analysis after laparoscopic cholecystectomy, the 
finding of a carcinoma would have made a large impact on the patient. Even with further operations, the patient would not have a favorable prognosis, considering the rapid progression of this malignant tumor.

\section{Conclusion}

The indication for laparoscopic cholecystectomy for patients with nonconfirmatory findings, even if asymptomatic, should be individualized and weighed with the impact of a late detection of carcinoma and the risk factors of this neoplasm to determine whether or not a surgical intervention is warranted.

\section{References}

1 - Sermon A, Himpens J, Leman G. Symptomatic adenomyomatosis of the gallbladder-report of a case. Acta Chir Belg. 2003;103(2):225-229. doi:10.1080/00015458.2003.11679412

2 - Golse N, Lewin M, Rode A, Sebagh M, Mabrut JY.

Gallbladder adenomyomatosis: Diagnosis and management. J Visc surg. 2017;154(5):345-353. doi:10.1016/j.jviscsurg.2017.06.004

3- Pellino G, Sciaudone G, Candilio G, et al. Stepwise approach and surgery for gallbladder adenomyomatosis: a minireview. Hepatobiliary Pancreat Dis Int. 2013;12(2):136-142. doi:10.1016/s14993872(13)60022-3
4 - Lin SH, Chang FY, Yang YS, Jin JS, Chen TW. Rare gallbladder adenomyomatosis presenting as atypical cholecystitis: case report. BMC Gastroenterol. 2011;11:106. Published 2011 Oct 5. doi:10.1186/1471-230X-11-106

5- Pais-Costa Sergio Renato, Farah José Francisco de Matos, Artigiani-Neto Ricardo, Franco Maria Isete Fares, Martins Sandro José, Goldenberg Alberto. Adenocarcinoma da vesícula biliar: avaliação dos fatores prognósticos em 100 casos ressecados no Brasil. ABCD, Arq. Bras. Cir. Dig. [Internet]. 2012 Mar [cited 2020 June 23] ; 25( 1 ): 13-19.

6 - Bonatti $M$, Vezzali N, Lombardo F, et al. Gallbladder adenomyomatosis: imaging findings, tricks and pitfalls. Insights Imaging. 2017;8(2):243-253. doi:10.1007/s13244-017-0544-7

7 - Nabatame N, Shirai $Y$, Nishimura A, Yokoyama N, Wakai I, Hatakeyama K. High risk of gallbladder carcinoma in elderly patients with segmental adenomyomatosis of the gallbladder. J Exp Clin Cancer Res. 2004;23:593-598.

8 - Hammad AY, Miura JT, Turaga KK, Johnston FM, Hohenwalter MD, Gamblin TC. A literature review of radiological findings to guide the diagnosis of gallbladder adenomyomatosis. HPB: The Official Journal of the International Hepato Pancreato Biliary Association. 2016;18(2):129-135. doi:10.1016/j.hpb.2015.09.006. 